\section{\$4. Detection System for Propagating Normal-zones with Pick-up Coils in the LHD Helical Coils}

Imagawa, S., Yanagi, N., Mito, T.

Propagation and recovery of normal zones were observed several times in a pair of helical coils, which are $\mathrm{H} 1$ and $\mathrm{H} 2$ coils, of the Large Helical Device. In order to detect the position of a propagating normal zone, pick-up coils were installed along the helical coils by the pitch of 30 degree of the poloidal angle, as shown in Fig. 1. They detect the change of magnetic field by current transfer between the superconducting strands and an aluminum stabilizer. The position and the velocity of propagating normal-zones were detected successfully 15 times since the pickup coils had been installed. They were induced 12 times in $\mathrm{H} 1$ coil and three times in $\mathrm{H} 2$ coil. Most of the normal zones were induced at the bottom of the coils, and all of them propagated to one side, which is the downstream of the transport current, with recovery from the opposite side. The propagation velocity can be estimated from the delay time of the peak voltage of the pickup coils. These obtained data are very useful to investigate the cause of the normal-zone propagation and cryogenic stability of the helical coils. Furthermore, this method is applicable for other superconducting magnets made of asymmetrical conductors.

Figure 2 shows the voltage drop, which is proportional to the overall resistance, of the conductor measured in a model coil. ${ }^{1)}$ The time constants for the decreasing resistance in propagating and recovering are 59 and $441 \mathrm{~ms}$, respectively. The time constant in recovering is obviously longer than the theoretical value which is four times of that in propagating. Its possible reason is that the high resistive layer of $\mathrm{Cu}-2 \% \mathrm{Ni}$ delays the current transfer from the aluminum. Since the joule loss is proportional to the square of the current density, the primary time constants for the current shift can be set at $\tau_{1}=118 \mathrm{~ms}$ in propagating and at $\tau_{2}=882 \mathrm{~ms}$ in recovering by doubling those for the resistance. On the other hand, the primary time constant of current diffusion into a metal with the thickness, $d$ and resistivity, $\rho$ is given by $\pi^{2} \rho /\left(\mu \mathrm{d}^{2}\right)$. The $\tau_{1}$ is just same as that of the aluminum stabilizer with $d=5.1 \mathrm{~mm}$ and $\rho=0.028$ $\mathrm{n} \Omega \mathrm{m}$ at $4 \mathrm{~K}$ and $6 \mathrm{~T}$.

In the cases of one-side propagation, the recovery starts before the current deeply diffuses into the aluminum. The voltage drop can be fitted using the duration of propagation, $t_{\mathrm{r}}$. Using the fitting curves from the analysis on one-dimensional current diffusion, the current shift of the conductor is calculated as Fig. $3 .^{2)}$ In order to estimate the outputs of the pick-up coils by the current shift, the transport current in a conductor is modeled by a set of linecurrent elements. Their position in a selected conductor is defined as Fig. 3 for each time step using the measured propagation velocity. The magnetic field at the center of each pick-up coil is calculated using Biot-Savart law. The calculated results are in good agreement with the measured data. In the case of the 14 th propagation at $11.30 \mathrm{kA}$, the best accordance is obtained in the case of $t_{\mathrm{r}}=0.08 \mathrm{~s}$ for the "L1T9" that is the last turn in the first layer. ${ }^{2)}$ Further analyses are needed to validate the conductor in which a normal zone propagated.

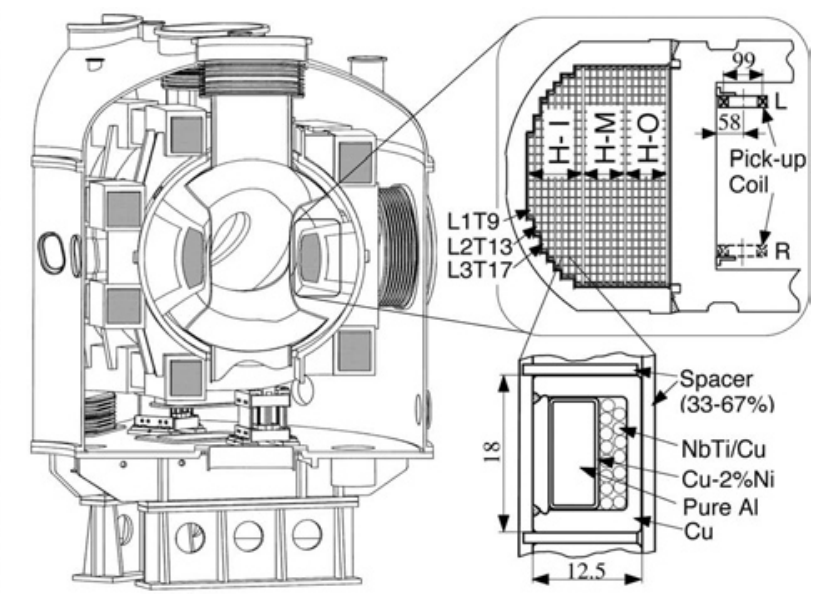

Fig. 1. Cross-section of the LHD cryostat and the helical coils, which consist of H-I, H-M, and H-O blocks from the inner side. The turn number of each block is 150 .

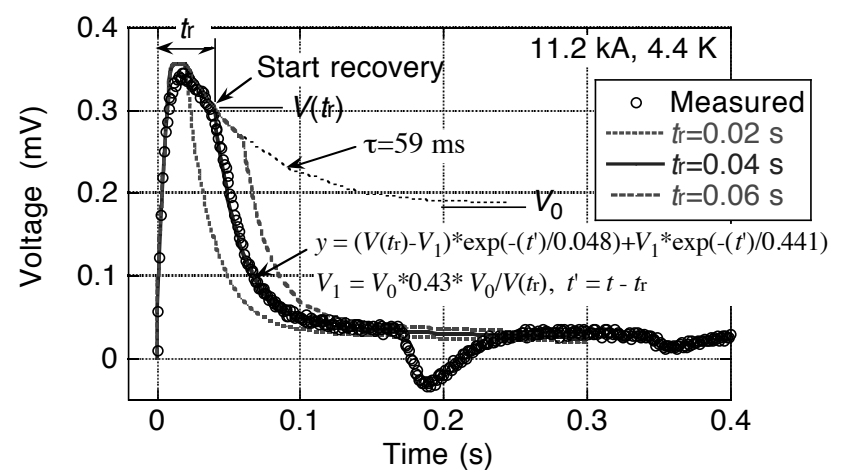

Fig. 2. Voltage of the LHD-HC conductor during propagation of a normal zone and recovery at $11.2 \mathrm{kA}$.

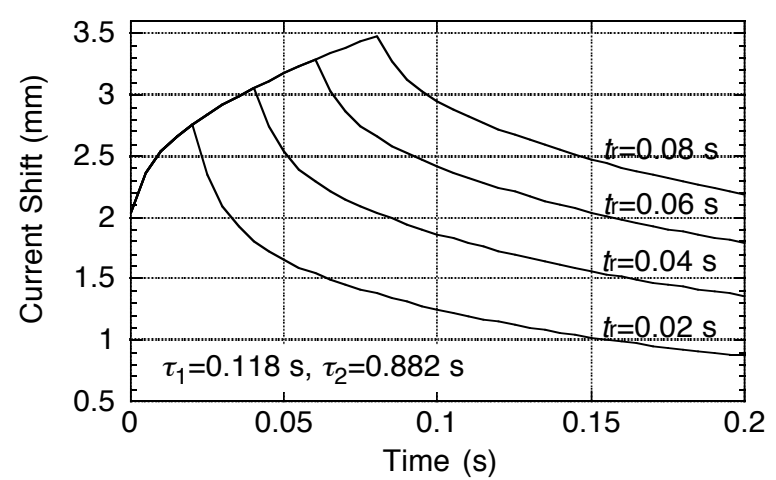

Fig. 3. The current shift of the conductor for the cases of the duration of propagation, $t_{\mathrm{r}}=0.02,0.04,0.06$, and $0.08 \mathrm{~s}$.

1) S. Imagawa, et al.: IEEE Trans. Appl. Supercond., vol. 14 (June 2004) 1388-1393.

2) S. Imagawa, et al.: IEEE Trans. Appl. Supercond., vol. 21 (June 2011) 2316-2319. 